\title{
Quadriceps-sparing versus traditional medial parapatellar approaches for total knee arthroplasty: a meta-analysis
}

\author{
Fu-Zhen Yuan, Ji-Ying Zhang, Dong Jiang and Jia-Kuo Yu*
}

\begin{abstract}
Background: There is still controversy regarding whether Quadriceps-sparing (QS) approach for total knee arthroplasty (TKA) lead to better earlier recovery as well as compromising low limb alignment and prosthesis position compared with conventional medial parapatellar (MP) approach. To overcome the shortcomings and inaccuracies of single studies, the clinical outcomes and radiographic assessments of QS approach and MP approach were evaluated through meta-analysis.

Methods: We performed this meta-analysis according to the Preferred Reporting Items for Systematic Reviews and Meta-analysis guidelines. A literature search was conducted in the PubMed, EMBase, Cochrane Collaboration Library and Web of Science databases. Our search strategy followed the requirements of the Cochrane Library Handbook. The study selection, data extraction and assessment of methodological quality were independently completed by four authors. And subgroup analysis and publication bias were also performed in the study.

Results: Eight prospective randomized controlled trials (RCTs) and eight retrospective studies were identified. Overall meta-analysis and subgroup meta-analysis of RCTs identified the QS approach mainly was associated with increased Knee Society function score beyond 24 months postoperatively (weighted mean difference [WMD] 1.78, $P=0.0004$ ) (WMD 1.86, $P=0.0002$ ), and improved range of motion $1-2$ weeks postoperatively (WMD 5.84, $P<0.00001)$ (WMD 4.87, $P=0.002$ ). Besides, lower visual analogue scale on postoperative day 1 (WMD $-0.91, P=0.02$ ), shorter hospital stay (WMD -0.88, $P=0.02$ ) and shorter incision (extension) (WMD -4.62, $P<0.00001$ ) were indicated in overall meta-analysis. However, surgical and tourniquet time was significantly longer in QS group by both overall and subgroup meta-analysis.

Conclusions: QS approach may accelerate early recovery without increasing the risk of malalignment of low limb and malposition of prosthesis.
\end{abstract}

Keywords: Knee arthroplasty, Meta-analysis, Minimally invasive, Quadriceps-sparing, Medial parapatellar

\section{Background}

Total knee arthroplasty (TKA) was first performed in 1968 [1]. It is widely used in patients with symptomatic, end-stage knee arthritis [2-4] and is the most successful surgical procedure for relieving pain and improving poor function in patients with advanced arthritis $[5,6]$. The conventional medial parapatellar (MP) approach has been established as the gold standard technique for TKA [7-11]. However, since the first quadriceps-sparing (QS) approach was performed in 2002 [12], it has become

\footnotetext{
*Correspondence: yujiakuo@126.com

Institution of Sports Medicine, Beijing Key Laboratory of Sports Injuries,

Peking University Third Hospital, Beijing 100191, China
}

one of the most common alternatives to the MP approach and, theoretically, provides a faster recovery of muscle. By avoiding violation of the extensor mechanism and suprapatellar pouch and everting the patella, the QS approach aims to produce less discomfort, provide a faster recovery and reduce the extent of patellar devascularization that can lead to patellar subluxation, dislocation, avascular necrosis, fracture, patellar component loosening, and anterior knee pain [13]. Currently, numerous well-designed studies have compared the outcomes of the QS and MP approaches. However, the conclusions from studies are still controversial. Some studies have found no significant differences between the two approaches $[14,15]$, whereas others

(c) The Author(s). 2019 Open Access This article is distributed under the terms of the Creative Commons Attribution 4.0 International License (http://creativecommons.org/licenses/by/4.0/), which permits unrestricted use, distribution, and reproduction in any medium, provided you give appropriate credit to the original author(s) and the source, provide a link to the Creative Commons license, and indicate if changes were made. The Creative Commons Public Domain Dedication waiver (http://creativecommons.org/publicdomain/zero/1.0/) applies to the data made available in this article, unless otherwise stated. 
have supported either the QS [16-23] or the MP approach [24-26]. Therefore, we designed this meta-analysis to quantitatively compare the efficacy and safety of the QS versus the MP approach for TKA.

\section{Methods}

Our meta-analysis was conducted according to the Preferred Reporting Items for Systematic Reviews and Meta-analysis (PRISMA) statement that established procedures for rigorous performance and reporting of meta-analyses $[27,28]$.

\section{Search strategy}

Two authors independently carried out a systematic search (last update 4 August 2018) of the PubMed, EMBase, Cochrane Collaboration Library and Web of Science databases, without restrictions on regions, publication types, or languages. The following search strategies were used in the search: \#1. (knee arthroplasty) OR knee replacement; \#2. (((((quadriceps-sparing) OR quadriceps sparing) OR quad-sparing) OR quad sparing) OR minimally invasive) OR mini-incision; \#3. \#1 AND \#2. Furthermore, the references from all accessed papers were also searched for any undetected studies. The results of our database search were imported into EndNote X7 and duplicates were eliminated using the duplicate removal function. Then, two authors screened all entries by title and abstract, and the remaining studies underwent full text review.

\section{Inclusion and exclusion criteria}

Studies were selected on the basis of the following criteria: (1) study design: randomized controlled trials (RCTs), and retrospective comparative studies (both cohort and case-control studies); (2) study population: adult patients who underwent primary TKA; (3) intervention: including both QS TKA and MP TKA; (4) available mean and standard deviation (SD) or proportion (or ability to estimate SD using data range). Review articles, case reports, editorials, letters to the editor, animal experimental studies and cadaver studies were excluded.

\section{Data extraction and methodological quality assessment}

Data were extracted using a predesigned sheet that included authors, publication data, specific interventions, main participant characteristics and results by three authors. Unreported data needed for this meta-analysis were obtained by communicating with the author though e-mail. For methodological quality evaluation of RCTs, recommendations issued by the Cochrane Handbook for Systematic Reviews were utilized in the meta-analysis [29]. The methodological quality of the included nonRCTs were evaluated with the modified Newcastle-Ottawa Scale (NOS), a simple tool used for the assessment of case controlled and cohort studies [30] that has been recommended by Cochrane collaboration [29]. NOS consists of three factors: patient selection, comparability of the study group and assessment of outcomes. According to NOS, a study can be awarded 0-9 stars.

\section{Statistical analysis}

This meta-analysis was performed with Review Manager 5.3 (Cochrane Collaboration, Oxford, UK). The level of significance was set at $P<0.05$. For dichotomous outcomes, the odds ratio (OR) and 95\% confidence interval $(95 \% \mathrm{CI})$ were calculated. For continuous outcomes, weighted mean difference (WMD) and the 95\% CI were calculated. Statistical heterogeneity was tested with the $I^{2}$ statistic and the Chi-squared $\left(\chi^{2}\right)$ test. A $P>0.1$ and an $I^{2} \leq 50 \%$ were considered to represent the absence of statistical heterogeneity. If significant heterogeneity $\left(I^{2}>50 \%\right)$ was found in the meta-analysis, a random effects model was used, otherwise, a fixed effects model was employed [29]. Certain studies in this meta-analysis provided data ranges (maximum and minimum values) rather than SDs. In these instances, SD was estimated as the difference between the maximum and minimum values divided by four [31], which serves as a conservative estimate of SD. Sensitivity analyses were conducted on the different types of study designs and the different participants enrolled in studies. Funnel plots were used to screen for potential publication bias.

\section{Results}

\section{Literature search}

The details of identifying relevant studies are shown in a flow chart of the study selection process (Fig. 1). The initial search identified 2038 potentially relevant citations from PubMed, EMBase, Cochrane Collaboration Library and Web of Science. After the duplicates were removed, 1903 studies were identified. A total of 1824 records were excluded based on a review of abstracts, leaving 79 articles for full-text review. Following full-text review, 16 citations were finally included consisting of eight RCTs $[15,16,21,24,25,32-34]$ and eight non-RCTs [14, 17-20, 23, 26, 35].

\section{The characteristics of included studies}

Table 1 summarizes the key characteristics of the included studies. There was a total of 1112 patients with 1439 TKAs in the included studies. The mean age of the included patients ranged from 42 to 88 years, the mean BMI ranged from 17.9 to $49 \mathrm{~kg} / \mathrm{m}^{2}$, and the mean follow-up duration ranged from 0 days to more than five years. Seven studies favored the QS approach results, while nine studies favored the MP approach results.

\section{Methodological quality assessment}

The quality assessment of the included studies is shown in Table 2, and methodological quality was regarded as high. 


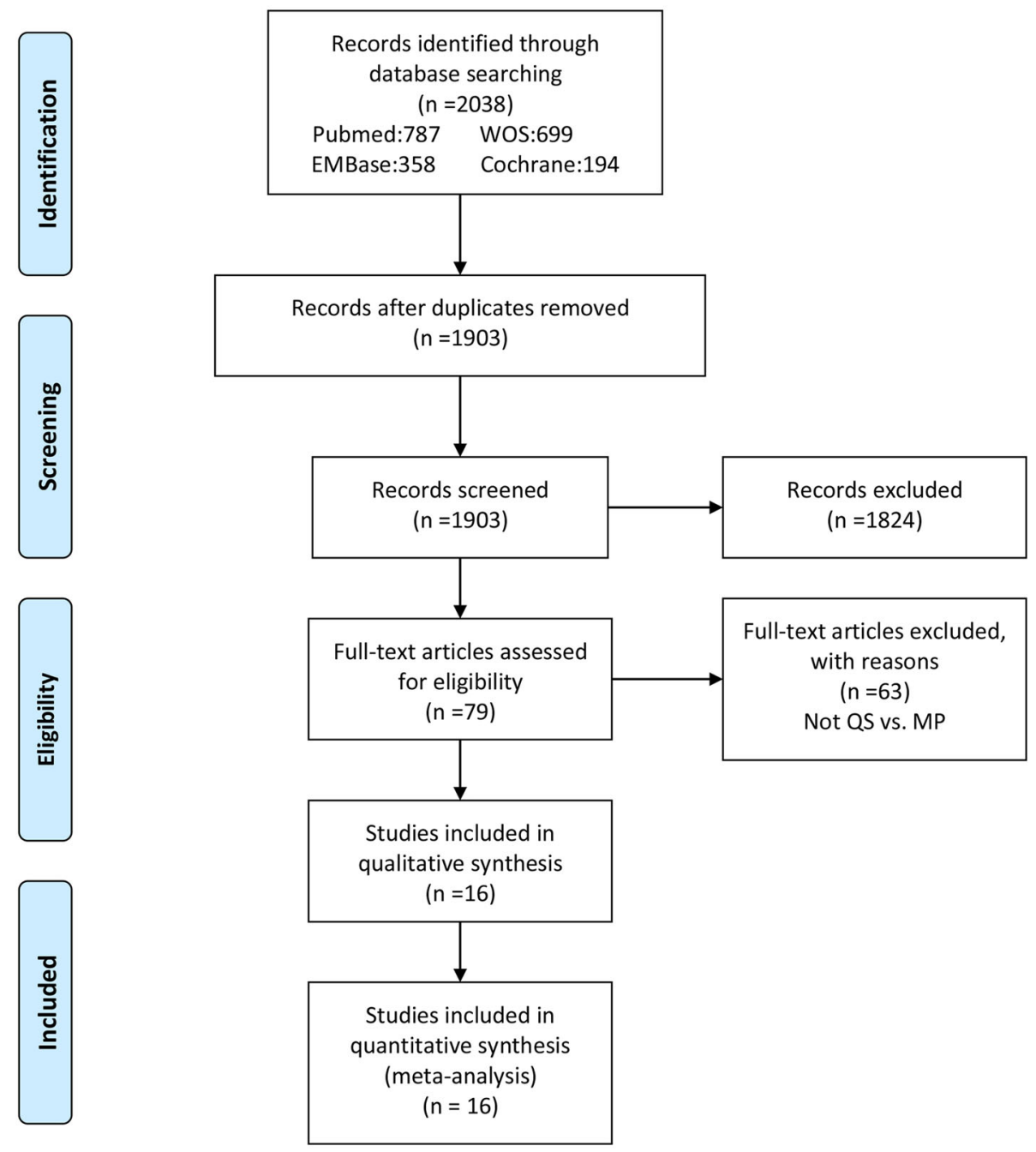

Fig. 1 PRISMA flow chart of literature screening

All eight RCTs were randomized, of which three RCTs utilized allocation concealment, four were blinded to participants and personnel, and five were blinded to outcome assessment. All the studies had incomplete data outcomes, and three selectively reported data. Observational studies achieving stars ranged from seven to eight points according to the Newcastle-Ottawa Scale, the total being nine points.

\section{Quantitative data synthesis}

There were 16 studies included for meta-analysis in which there were eight RCTs [15, 16, 21, 24, 25, 32-34] and eight non-RCTs [14, 17-20, 23, 26, 35].

\section{Primary outcomes}

The overall meta-analysis results (Table 3) were in favor of the QS approach based on long-term Knee Society (KS) function score (WMD 1.78, 95\% CI 0.80 to $2.76, P$ $\left.=0.0004, I^{2}=0 \%\right)$. Furthermore, the results showed that there were no significant differences between the QS and MP approaches in the KS Knee Score beyond 24 months postoperatively (WMD $-0.02,95 \%$ CI -0.69 to $0.65, P=0.95, I^{2}=0 \%$ ), in range of motion (ROM) beyond 16 months postoperatively (WMD $0.08,95 \% \mathrm{CI}-1.40$ to 1.57, $P=0.91, I^{2}=4 \%$ ), or complications (OR $0.87,95 \% \mathrm{CI}$ 0.49 to $1.54, P=0.63, I^{2}=9 \%$ ), infections (OR $1.53,95 \% \mathrm{CI}$ 0.69 to $3.39, P=0.29, I^{2}=0 \%$ ), mechanical axis outliers (OR $1.05,95 \%$ CI 0.65 to $1.72, P=0.83, I^{2}=27 \%$ ), femoral component coronal angle outliers (OR 2.30, 95\% CI 0.35 to $\left.15.24 P=0.39, I^{2}=65 \%\right)$, tibial component coronal angle outliers (OR $0.73,95 \%$ CI 0.40 to $1.33, P=0.30, I^{2}=$ $40 \%$ ), mechanical axis (WMD $0.35,95 \%$ CI -0.02 to 0.73 , $\left.P=0.07, I^{2}=0 \%\right)$, femoral component coronal angle (WMD $0.23,95 \% \mathrm{CI}-0.90$ to $1.35, P=0.69, I^{2}=92 \%$ ), tibial component coronal angle (WMD -0.40, 95\% CI -1.29 to $0.49, P=0.38, I^{2}=92 \%$ ), lateral patellar tilt (WMD $-1.25,95 \% \mathrm{CI}-3.36$ to $\left.0.85, P=0.24, I^{2}=78 \%\right)$ or lateral 
Table 1 Characteristics of included studies

\begin{tabular}{|c|c|c|c|c|c|c|c|c|c|}
\hline Study/Year & Country & $\begin{array}{l}\text { Recruitment } \\
\text { period }\end{array}$ & Group & $\begin{array}{l}\text { Patients } \\
\text { (male/female) }\end{array}$ & $\begin{array}{l}\text { Number of } \\
\text { TKAs }\end{array}$ & Age (year) & BMI $\left(\mathrm{kg} / \mathrm{m}^{2}\right)$ & $\begin{array}{l}\text { Follow-up } \\
\text { (months) }\end{array}$ & $\begin{array}{l}\text { Results } \\
\text { Favor }\end{array}$ \\
\hline \multirow[t]{2}{*}{ Huang 2016} & \multirow[t]{2}{*}{ China } & \multirow[t]{2}{*}{$2005-2007$} & QS & $2 / 29$ & 31 & $69.3 \pm 7.9$ & $26.9 \pm 3.3$ & $65 \pm 3.8$ & \multirow[t]{2}{*}{ QS } \\
\hline & & & MP & $2 / 28$ & 30 & $71.2 \pm 5.8$ & $26.7 \pm 2.8$ & $68 \pm 5.4$ & \\
\hline \multirow[t]{2}{*}{ Qi 2016} & \multirow[t]{2}{*}{ China } & \multirow[t]{2}{*}{$2005-2007$} & QS & $2 / 26$ & 30 & $65.3 \pm 6.9$ & $26.5 \pm 3.0$ & 74.8 & \multirow[t]{2}{*}{ QS } \\
\hline & & & MP & $2 / 24$ & 28 & $64.0 \pm 5.7$ & $28.1 \pm 4.1$ & 74.8 & \\
\hline \multirow[t]{2}{*}{ Lin 2013} & \multirow[t]{2}{*}{ Taiwan } & \multirow[t]{2}{*}{$2007-2008$} & QS & $5 / 30$ & 35 & $67.7(60,78)$ & $26.3(21.2,29.7)$ & 24 & \multirow[t]{2}{*}{ MP } \\
\hline & & & $\mathrm{MP}$ & $5 / 30$ & 35 & $68.5(55,77)$ & $25.9(20,29.5)$ & 24 & \\
\hline \multirow[t]{2}{*}{ Xu 2013} & \multirow[t]{2}{*}{ China } & \multirow[t]{2}{*}{ 2009-2010 } & QS & $7 / 19$ & 35 & $63.5 \pm 8.7$ & $25.2 \pm 3.4$ & 24 & \multirow[t]{2}{*}{ QS } \\
\hline & & & MP & $11 / 18$ & 35 & $64.2 \pm 9.3$ & $25.2 \pm 2.3$ & 24 & \\
\hline \multirow[t]{2}{*}{ Chiang 2012} & \multirow[t]{2}{*}{ Taiwan } & \multirow[t]{2}{*}{2005} & QS & $3 / 27$ & 38 & $69.7 \pm 5.3$ & $28.6 \pm 3.8$ & 24 & \multirow[t]{2}{*}{ MP } \\
\hline & & & MP & $3 / 27$ & 37 & $69.8 \pm 5.4$ & $29.6 \pm 3.5$ & 24 & \\
\hline \multirow{2}{*}{$\begin{array}{l}\text { Matsumoto } \\
2011\end{array}$} & \multirow[t]{2}{*}{ Japan } & \multirow[t]{2}{*}{ 2005-2007 } & QS & $0 / 25$ & 25 & $73.8 \pm 1.7$ & Unclear & 0 & \multirow[t]{2}{*}{ MP } \\
\hline & & & MP & $0 / 25$ & 25 & $73.7 \pm 1.4$ & Unclear & 0 & \\
\hline \multirow[t]{2}{*}{ Yang 2010} & \multirow[t]{2}{*}{ Korea } & \multirow[t]{2}{*}{$2006-2007$} & QS & $1 / 14$ & 24 & $66.7 \pm 6.9$ & Unclear & 24 & \multirow[t]{2}{*}{ MP } \\
\hline & & & MP & $2 / 14$ & 23 & $68 \pm 6.8$ & Unclear & 24 & \\
\hline Karpman 2009 & USA & 2004-2005 & QS & $8 / 12$ & 20 & $73 \pm 7.4$ & $28 \pm 4.4$ & 6 & QS \\
\hline & & & MP & $9 / 10$ & 19 & $73 \pm 5.1$ & $29 \pm 4.6$ & 6 & \\
\hline Chotanaphuti & Thailand & 2004-2005 & QS & $3 / 17$ & 20 & $68.4(58,78)$ & Unclear & 0.25 & QS \\
\hline & & & MP & $4 / 16$ & 20 & $67.5(56,80)$ & Unclear & 0.25 & \\
\hline Shen 2007 & China & $2005-2006$ & QS & Unclear & 26 & Unclear & Unclear & 17 & QS \\
\hline & & & MP & Unclear & 33 & Unclear & Unclear & 17 & \\
\hline Huang 2007 & Taiwan & 2004-2005 & QS & $6 / 26$ & 32 & $63(56,72)$ & Unclear & 24 & MP \\
\hline & & & MP & $7 / 28$ & 35 & $65(59,75)$ & Unclear & 24 & \\
\hline King 2007 & USA & 2003-2005 & QS & $48 / 52$ & 100 & $67(44,84)$ & $30(22,43)$ & 1.5 & QS \\
\hline & & & MP & $17 / 28$ & 45 & $66(42,85)$ & $32(20,49)$ & 1.5 & \\
\hline Kim 2007 & Korea & 2004-2005 & QS & $27 / 93$ & 120 & $65.4(43,88)$ & $28.1(19,36)$ & 21.5 & MP \\
\hline & & & MP & $27 / 93$ & 120 & $65.4(43,88)$ & $28.1(19,36)$ & 21.5 & \\
\hline Chin 2007 & Singapore & 2004 & QS & $6 / 24$ & 30 & $69.0(57,80)$ & $27.53(18.6,34.2)$ & Unclear & MP \\
\hline & & & MP & $3 / 27$ & 30 & $63.4(47,80)$ & $29.44(22.7,40)$ & Unclear & \\
\hline Chen 2006 & USA & Prior to 2002 & QS & $11 / 17$ & 32 & $70(50,86)$ & $28.5(17.9,39.9)$ & 33 & MP \\
\hline & & & MP & $11 / 18$ & 38 & $67(42,81)$ & $28.7(21.6,40.1)$ & 40 & \\
\hline Kim 2006 & Korea & 2003 & QS & $7 / 65$ & 144 & $68.6(57,85)$ & 27.2 & 13.6 & MP \\
\hline & & & MP & $8 / 64$ & 144 & $67.4(58,84)$ & 28.1 & 13.6 & \\
\hline
\end{tabular}

TKA total knee arthroplasty, BMI body mass index, QS quadriceps-sparing, MP medial parapatellar

patellar displacement (WMD $-1.47,95 \%$ CI -4.59 to 1.66 , $P=0.36, I^{2}=90 \%$ ).

\section{Secondary outcomes}

Meta-analysis showed that, when compared with the MP approach, the QS approach significantly improved ROM 1-2 weeks postoperatively (WMD 5.84, 95\% CI 3.84 to 7.83, $P<0.00001, I^{2}=21 \%$ ), shortened length of stay (WMD $-0.88,95 \%$ CI -1.62 to $-0.15, P=0.02, I^{2}=94 \%$ ) and reduced the length of incision in extension (WMD $-4.62,95 \%$ CI -6.35 to $\left.-2.90, P<0.00001, I^{2}=99 \%\right)$. However, the QS approach significantly increased surgical time (WMD 12.02, 95\% CI 4.06 to $19.98, P=$ $0.003, I^{2}=95 \%$ ) and tourniquet time (WMD 27.19, 95\% CI 9.17 to $\left.45.22, P=0.003, I^{2}=99 \%\right)$. Although the meta-analysis demonstrated significant differences in visual analogue scale (VAS) on postoperative day 1 (WMD $-0.91,95 \%$ CI -1.68 to $\left.-0.41, P=0.02, I^{2}=81 \%\right)$. No other significant differences were found for secondary outcomes as shown in Table 4.

\section{Subgroup analysis}

A pooling of the RCTs is summarized in Table 5. The QS approach extended the surgical time (WMD 18.86, 
Table 2 Quality assessment of included studies

\begin{tabular}{|c|c|c|c|c|c|c|c|}
\hline Study/Year & $\begin{array}{l}\text { Random Sequence } \\
\text { Generation }\end{array}$ & $\begin{array}{l}\text { Allocation } \\
\text { Concealment }\end{array}$ & $\begin{array}{l}\text { Blinding of Participants } \\
\text { and Personnel }\end{array}$ & $\begin{array}{l}\text { Blinding of Outcome } \\
\text { Assessment }\end{array}$ & $\begin{array}{l}\text { Incomplete } \\
\text { Outcome Data }\end{array}$ & $\begin{array}{l}\text { Selective } \\
\text { Reporting }\end{array}$ & $\begin{array}{l}\text { Other } \\
\text { Bias }\end{array}$ \\
\hline $\operatorname{Lin} 2013^{*}$ & Yes & $\begin{array}{l}\text { Sealed } \\
\text { envelope }\end{array}$ & Unclear & Yes & Yes & Unclear & Unclear \\
\hline Xu $2013^{*}$ & Yes & $\begin{array}{l}\text { Sealed } \\
\text { envelope }\end{array}$ & Unclear & Unclear & Yes & Unclear & Unclear \\
\hline $\begin{array}{l}\text { Chiang } \\
2012^{*}\end{array}$ & Yes & Unclear & Yes & Yes & Yes & Yes & Unclear \\
\hline $\begin{array}{l}\text { Matsumoto } \\
2011^{*}\end{array}$ & Yes & Unclear & Yes & Unclear & Yes & Unclear & Unclear \\
\hline Yang $2010^{*}$ & Yes & Unclear & Unclear & Yes & Yes & Yes & Unclear \\
\hline $\begin{array}{l}\text { Karpman } \\
2009^{*}\end{array}$ & Yes & Unclear & Yes & Yes & Yes & Unclear & Unclear \\
\hline Kim $2007^{*}$ & Yes & Unclear & Unclear & Yes & Yes & Unclear & Unclear \\
\hline \multirow[t]{2}{*}{ Chin $2007^{*}$} & Yes & $\begin{array}{l}\text { Sealed } \\
\text { envelope }\end{array}$ & Yes & Yes & Yes & Yes & Unclear \\
\hline & & Selection & Comparability & & Outcomes & & $\begin{array}{l}\text { Total } \\
\text { score }\end{array}$ \\
\hline \multicolumn{2}{|l|}{ Huang $2016^{\dagger}$} & 2 & 2 & & 3 & & 7 \\
\hline \multicolumn{2}{|l|}{ Qi $2016^{\dagger}$} & 3 & 2 & & 2 & & 7 \\
\hline \multicolumn{2}{|c|}{ Chotanaphuti $2008^{\dagger}$} & 3 & 2 & & 3 & & 8 \\
\hline \multicolumn{2}{|l|}{ Shen $2007^{\dagger}$} & 3 & 2 & & 3 & & 8 \\
\hline \multicolumn{2}{|l|}{ Huang $2007^{\dagger}$} & 2 & 2 & & 3 & & 7 \\
\hline \multicolumn{2}{|l|}{ King $2007^{\dagger}$} & 3 & 2 & & 3 & & 8 \\
\hline \multicolumn{2}{|l|}{ Chen $2006^{\dagger}$} & 3 & 2 & & 2 & & 7 \\
\hline \multicolumn{2}{|l|}{ Kim $2006^{\dagger}$} & 3 & 2 & & 3 & & 8 \\
\hline
\end{tabular}

${ }^{*}$ The risk of bias was assessed independently using the Cochrane Handbook for Systematic Reviews of Interventions; ${ }^{\dagger}$ Methodological quality of the included studies was assessed according to Newcastle-Ottawa Scale

Table 3 Primary outcomes of meta-analysis results

\begin{tabular}{|c|c|c|c|c|c|c|}
\hline Outcomes of Demographics & $\begin{array}{l}\text { Number of Contributing } \\
\text { Studies }\end{array}$ & $\begin{array}{l}\text { Number of QS } \\
\text { TKAs }\end{array}$ & $\begin{array}{l}\text { Number of MP } \\
\text { TKAs }\end{array}$ & $\begin{array}{l}\text { WMD or OR }(95 \% \\
\text { Cl) }\end{array}$ & $\begin{array}{l}P- \\
\text { Value }\end{array}$ & Heterogeneity \\
\hline KS Knee Score beyond 24 months & 4 & 330 & 329 & $-0.02(-0.69,0.65)$ & 0.95 & $0 \%$ \\
\hline $\begin{array}{l}\text { KS Function Score beyond } 24 \\
\text { months }\end{array}$ & 3 & 186 & 185 & $1.78(0.80,2.76)$ & 0.0004 & $0 \%$ \\
\hline ROM beyond 16 months & 6 & 400 & 404 & $0.08(-1.40,1.57)$ & 0.91 & $4 \%$ \\
\hline Complications & 10 & 464 & 430 & $0.87(0.49,1.54)$ & 0.63 & $9 \%$ \\
\hline Infections & 10 & 503 & 515 & $1.53(0.69,3.39)$ & 0.29 & $0 \%$ \\
\hline Mechanical axis outliers & 5 & 257 & 266 & $1.05(0.65,1.72)$ & 0.83 & $27 \%$ \\
\hline $\begin{array}{l}\text { Femoral component coronal angle } \\
\text { outliers }\end{array}$ & 4 & 237 & 235 & $2.30(0.35,15.24)$ & 0.39 & $65 \%$ \\
\hline $\begin{array}{l}\text { Tibial component coronal angle } \\
\text { outliers }\end{array}$ & 5 & 337 & 280 & $0.73(0.40,1.33)$ & 0.30 & $40 \%$ \\
\hline Mechanical axis & 5 & 149 & 147 & $0.35(-0.02,0.73)$ & 0.07 & $0 \%$ \\
\hline Femoral component coronal angle & 6 & 395 & 395 & $0.23(-0.90,1.35)$ & 0.69 & $92 \%$ \\
\hline Tibial component coronal angle & 7 & 495 & 445 & $-0.40(-1.29,0.49)$ & 0.38 & $92 \%$ \\
\hline Lateral patellar tilt & 5 & 418 & 363 & $-1.25(-3.36,0.85)$ & 0.24 & $78 \%$ \\
\hline Lateral patellar displacement & 2 & 131 & 75 & $-1.47(-4.59,1.66)$ & 0.36 & $90 \%$ \\
\hline
\end{tabular}


Table 4 Secondary outcomes of meta-analysis results

\begin{tabular}{lllllll}
\hline Outcomes of Demographics & Number of Contributing Studies & $\begin{array}{l}\text { Number of } \\
\text { QS TKAs }\end{array}$ & $\begin{array}{l}\text { Number of } \\
\text { MP TKAs }\end{array}$ & WMD or OR (95\% Cl) & $P$ - Value & Heterogeneity \\
\hline KS Knee Score 1.5-3 months & 4 & 204 & 212 & $1.27(-0.57,3.11)$ & 0.18 & $57 \%$ \\
KS Function Score 1.5-3 months & 4 & 204 & 212 & $-0.09(-3.98,3.81)$ & 0.97 & $73 \%$ \\
ROM 1-2 weeks & 5 & 148 & 162 & $5.84(3.84,7.83)$ & $<0.00001$ & $21 \%$ \\
ROM 4-8 weeks & 7 & 283 & 247 & $0.51(-1.90,2.91)$ & 0.68 & $61 \%$ \\
ROM 3 months & 2 & 152 & 158 & $-0.60(-2.32,1.12)$ & 0.50 & $47 \%$ \\
ROM 12 months & 2 & 58 & 68 & $4.00(-5.80,13.80)$ & 0.42 & $90 \%$ \\
VAS 1 day & 6 & 183 & 197 & $-0.91(-1.68,-0.41)$ & 0.02 & $81 \%$ \\
VAS 3 days & 2 & 64 & 70 & $-0.93(-2.01,0.14)$ & 0.09 & $82 \%$ \\
VAS 4-8 weeks & 3 & 84 & 89 & $-0.26(-1.13,0.61)$ & 0.56 & $77 \%$ \\
Surgical time (min) & 8 & 507 & 450 & $12.02(4.06,19.98)$ & 0.003 & $95 \%$ \\
Tourniquet time (min) & 8 & 447 & 462 & $27.19(9.17,45.22)$ & 0.003 & $99 \%$ \\
Intraoperative blood loss (ml) & 4 & 334 & 339 & $1.99(-14.28,18.25)$ & 0.81 & $0 \%$ \\
Total blood loss (ml) & 5 & 255 & 254 & $-42.94(-150.57,64.70)$ & 0.43 & $90 \%$ \\
Incision, extension (cm) & 7 & 276 & 284 & $-4.62(-6.35,-2.90)$ & $<0.00001 \quad 99 \%$ \\
Incision, flexion (cm) & 3 & 193 & 192 & $-1.90(-3.99,0.19)$ & 0.07 & $99 \%$ \\
Length of stay (days) & 8 & 433 & 441 & $-0.88(-1.62,-0.15)$ & 0.02 & $94 \%$ \\
SLR at 24h (\% of patients) & 3 & 105 & 107 & $3.05(0.89,10.53)$ & 0.08 & $75 \%$ \\
\hline VAS, visua & & & &
\end{tabular}

VAS, visual analogue scale; SLR, straight leg rising

95\% CI 8.81 to $\left.28.91, P=0.0002, I^{2}=94 \%\right)$ and tourniquet time (WMD 24.39, 95\% CI 3.19 to $45.60, P=0.02$, $\left.I^{2}=99 \%\right)$. However, the QS approach significantly improved ROM 1-2 weeks postoperatively (WMD 4.87, 95\% CI 1.78 to $9.76, P=0.002, I^{2}=0 \%$ ) and shortened the incision scar in extension (WMD -3.76, 95\% CI -6.79 to $\left.-0.73, P=0.02, I^{2}=99 \%\right)$. Furthermore, the meta-analysis of RCTs also showed that the QS approach was associated with a higher KS Function Score beyond 24 months postoperatively (WMD $1.86,95 \%$ CI 0.86 to $2.85, P=0.0002, I^{2}=0 \%$ ).

\section{Publication bias}

Figure 2 shows a funnel plot of the studies included in this meta-analysis that reported infections. All studies lie inside the $95 \%$ CIs, with an even distribution around the vertical, indicating no obvious publication bias.

\section{Discussion}

The results suggest that QS approach may be associated with higher KS function score beyond 24 months postoperatively, could improve ROM 1-2 weeks postoperatively, and shorten incision (extension) with significantly longer surgical and tourniquet time in both overall and subgroup meta-analysis.

According to both the subgroup meta-analysis of RCTs and the overall meta-analysis, results showed that the QS approach was favored in terms of the KS function score beyond 24 months postoperatively which was a primary outcome with a WMD 1.78 and 1.86, respectively. However, we cautiously thought that QS approach could not be confirmed as superior because Lee et al. [36] found that a minimal clinically important difference (MCID) in the KS function score was between 6.1 and 6.4. Besides, QS approach significantly improved ROM 1-2 week postoperatively and shortened the incision length in extension. Longer surgical time and tourniquet time were needed in QS group without increasing complications and infections. Based on these results of the secondary outcomes, we identified that the QS approach may accelerate early recovery to some extent and improved cosmesis which may make patients to be more satisfied with their surgery without increasing the risk of surgery. But it is undeniable that a longer surgery time may lead to increased hospital costs.

In our overall meta-analysis, the QS approach had significant advantages over the MP approach on VAS 1 day postoperatively and length of stay, which were not identified in the subgroup meta-analysis of RCTs. Although the WMD was statistically significant, it falls below the threshold for clinical significance according to the MCID of VAS [37]. Therefore, the possibility that QS approach may accelerate early recovery was supported to a limited extent.

Meanwhile, we observed that the QS approach was not associated with a higher risk of malalignment of low limb and poor position of prosthesis, which was demonstrated in both overall and subgroup meta-analysis. The importance of accurate lower limb alignment and prothesis position after TKA and the greater risk of implant failure with malalignment have been well recognized [38, 39]. 
Table 5 Meta-analysis results of RCTs

\begin{tabular}{|c|c|c|c|c|c|c|}
\hline Outcomes of Demographics & $\begin{array}{l}\text { Number of Contributing } \\
\text { Studies }\end{array}$ & $\begin{array}{l}\text { Number of } \\
\text { QS TKAs }\end{array}$ & $\begin{array}{l}\text { Number of } \\
\text { MP TKAs }\end{array}$ & $\begin{array}{l}\text { WMD or OR } \\
(95 \% \mathrm{Cl})\end{array}$ & $P$ - Value & Heterogeneity \\
\hline KS Knee Score beyond 24 months & 2 & 155 & 155 & $-0.18(-1.13,0.77)$ & 0.71 & $25 \%$ \\
\hline KS Function Score beyond 24 months & 2 & 155 & 155 & $1.86(0.86,2.85)$ & 0.0002 & $0 \%$ \\
\hline ROM beyond 16 months & 3 & 193 & 192 & $-0.41(-2.18,1.37)$ & 0.65 & $0 \%$ \\
\hline Complications & 5 & 243 & 244 & $1.49(0.68,3.27)$ & 0.32 & $1 \%$ \\
\hline Infections & 7 & 301 & 300 & $1.95(0.75,5.10)$ & 0.17 & $0 \%$ \\
\hline Mechanical axis outliers & 2 & 55 & 54 & $3.80(0.61,23.57)$ & 0.15 & $25 \%$ \\
\hline $\begin{array}{l}\text { Femoral component coronal angle } \\
\text { outliers }\end{array}$ & 3 & 93 & 91 & $5.24(0.80,34.28)$ & 0.08 & $20 \%$ \\
\hline Tibial component coronal angle outliers & 3 & 93 & 91 & $4.14(0.87,19.75)$ & 0.07 & $0 \%$ \\
\hline Mechanical axis & 3 & 88 & 89 & $0.34(-0.37,1.05)$ & 0.35 & $15 \%$ \\
\hline Femoral component coronal angle & 5 & 251 & 251 & $0.07(-1.30,1.44)$ & 0.92 & $93 \%$ \\
\hline Tibial component coronal angle & 5 & 251 & 251 & $-0.31(-1.58,0.97)$ & 0.64 & $93 \%$ \\
\hline Lateral patellar tilt & 2 & 143 & 144 & $0.73(-0.30,1.76)$ & 0.16 & $0 \%$ \\
\hline KS Knee Score 1.5-3 months & 3 & 178 & 179 & $1.01(-0.74,2.76)$ & 0.26 & $61 \%$ \\
\hline KS Function Score 1.5-3 months & 3 & 178 & 179 & $-0.67(-5.45,4.10)$ & 0.78 & $82 \%$ \\
\hline ROM 1-2 weeks & 2 & 58 & 56 & $4.87(1.78,7.96)$ & 0.002 & $0 \%$ \\
\hline ROM 4-8 weeks & 3 & 93 & 91 & $1.68(-2.16,5.51)$ & 0.39 & $60 \%$ \\
\hline VAS 1 day & 3 & 93 & 91 & $-0.07(-0.49,0.35)$ & 0.74 & $0 \%$ \\
\hline VAS 4-8 weeks & 2 & 58 & 56 & $-0.46(-2.31,1.40)$ & 0.63 & $87 \%$ \\
\hline Surgical time (min) & 5 & 243 & 241 & $18.86(8.81,28.91)$ & 0.0002 & $94 \%$ \\
\hline Tourniquet time (min) & 3 & 193 & 192 & $24.39(3.19,45.60)$ & 0.02 & $99 \%$ \\
\hline Intraoperative blood loss (ml) & 2 & 158 & 157 & $3.10(-24.89,31.09)$ & 0.83 & $0 \%$ \\
\hline Total blood loss (ml) & 4 & 111 & 110 & $4.24(-56.29,64.77)$ & 0.89 & $48 \%$ \\
\hline Incision, extension (cm) & 4 & 188 & 188 & $-3.76(-6.79,-0.73)$ & 0.02 & $99 \%$ \\
\hline Incision, flexion (cm) & 3 & 193 & 192 & $-1.90(-3.99,0.19)$ & 0.07 & $99 \%$ \\
\hline Length of stay (days) & 4 & 205 & 204 & $-0.34(-1.02,0.34)$ & 0.33 & $71 \%$ \\
\hline SLR at $24 \mathrm{~h}$ (\% of patients) & 2 & 73 & 72 & $1.62(0.79,3.30)$ & 0.19 & $0 \%$ \\
\hline
\end{tabular}

TKA total knee arthroplasty, QS quadriceps-sparing, MP medial parapatellar, WMD weighted mean difference, $O R$ odds ratio, $\mathrm{Cl}$ confidence interval, $K S$ knee society, $R O M$ range of motion, VAS visual analogue scale, SLR straight leg rising

Owing to the importance of those factors, we should pay attention to this situation even though meta-analysis did not identify this issue. As arthroplasty surgeons know, the QS approach can easily be extended or converted to the MP approach during the surgery. Therefore, if a surgeon is not sufficiently skilled in the TKA procedure, the QS approach should be appropriately extended to ensure good bone resection and prosthesis installation.

The findings from our meta-analysis are in partial disagreement with the results and conclusions of two recent meta-analyses by Peng et al. [40] and Kazarian et al. [41]. The disagreements are not only due to differences in the concluded articles and the extraction and selection of data; they are also due to differences in the included articles of RCTs. In our view, the meta-analysis by Peng et al. included three studies that did not meet the inclusion criteria and excluded two articles that met the inclusion criteria. In the included studies of Peng et al., Shen et al. [18] was a cohort study, Tasker et al. [42] compared the mini-midvastus or subvastus approach to the MP approach and Lin et al. [43] compared the QS approach to the mini-MP approach. In addition, Peng et al. did not include two studies $[15,21]$ that met the inclusion criteria of the meta-analysis. For the meta-analysis by Kazarian et al., we considered that an article by Yang et al. [15] met the inclusion criteria even though it was not included and a study comparing the QS approach with the mini-MP approach by Lin et al. [43] was enrolled. Because these deviations could potentially affect some of the results, they might provide an explanation for the partial disagreement between our meta-analyses.

The inclusion of both RCTs and retrospective comparative studies enhanced the sample size and robustness of 


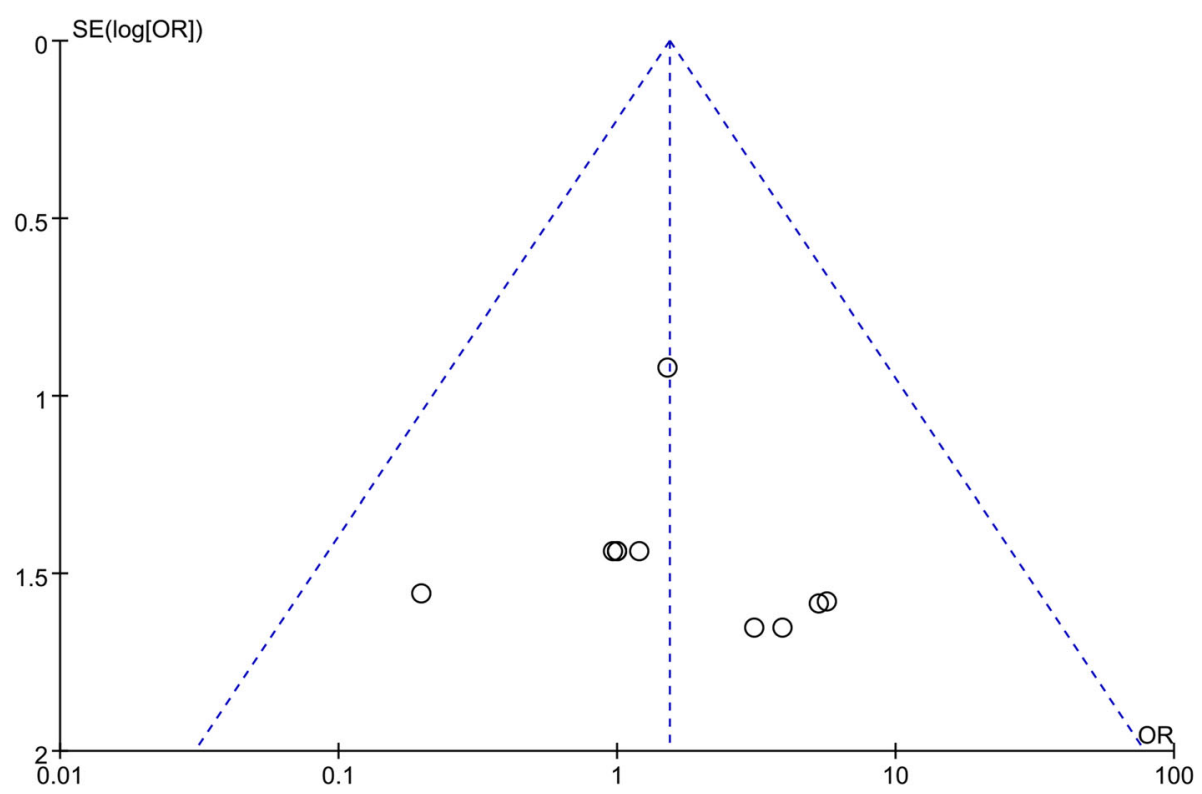

Fig. 2 Funnel plot illustrating meta-analysis of infections

the estimates when compared with previous studies [40, 41]. Although a meta-analysis of RCTs only would be ideal, the limited number of RCTs and their size limits the scope of this review and prevents its findings from being conclusive.

Between-study heterogeneity was found to exist with some outcomes. Included studies adopted different research objects, research designs, and measurement of results, differences, all of which may contribute to the significant between-study heterogeneity. After careful analysis of these documents, we found that design and objects were also potential contributors to heterogeneity. Therefore, we conducted a subgroup meta-analysis pooling only RCTs to increase the reference value of the results. It is well known that RCTs standardize the research process through randomization, blinding, strict quality control, etc. to obtain reliable research results. In addition, if heterogeneity persisted, we adopted random-effects model to potentially reduce, but not abolish, the effect of heterogeneity. The limitation is that the duration of follow-up of these studies is still not long enough. A follow-up period of more than ten years is required to evaluate and confirm outcomes, especially regarding relationships between the mechanical axis, prosthesis position and functional scores.

\section{Conclusions}

In summary, the use of QS approach in patients undergoing TKAs appears to be effective in improving ROM 1-2 postoperatively and reducing the length of incision in knee extension. In addition, the overall meta-analysis illustrated that QS approach may shorten the length of stay. However, the QS approach also significantly increases surgical and tourniquet time. Apart from this, the two surgical techniques appear to be equivalent in other aspects such as mechanical axis, prosthesis position, complications, infections and so on. On the basis of these findings, we are optimistic about the QS approach to some extent.

\section{Abbreviations}

BMI: body mass index; Cl: Confidence interval; DVT: Deep vein thrombosis; KS: Knee society; MIS-TKA: Minimally invasive surgery-total knee arthroplasty; MP TKA: Medial parapatellar total knee arthroplasty; NOS: Newcastle-Ottawa Scale; OR: Odds ratio; PRISMA: Preferred Reporting Items for Systematic Reviews and Meta-analysis; QS TKA: Quadriceps-sparing total knee arthroplasty; RCT: Randomized controlled trial; ROM: Range of motion; SLR: Straight leg raising; TKA: Total knee arthroplasty; VAS: Visual analog scale; WMD: Weighted mean difference

\section{Acknowledgements}

This work was supported by the National Key R\&D Program of China (2017YFB1303001). The funders had no role in study design, data collection and analysis, decision to publish, or preparation of the manuscript.

\section{Funding}

There is no funding source.

\section{Availability of data and materials}

The datasets used and/or analysed during the current study are available from the corresponding author on reasonable request.

\section{Authors' contributions}

Conception and design: FZY, DJ, JKY. Analysis and interpretation of the data: $F Z Y, J Y Z, D J$. Drafting of the article: FZY, DJ, JKY. Critical revision of the article for important intellectual content: DJ, JKY. Statistical expertise: FZY, JYZ. All authors read and approved the final manuscript.

Ethics approval and consent to participate Not applicable. 


\section{Consent for publication}

Not applicable.

\section{Competing interests}

The authors declare that they have no competing interests regarding the publication of this paper.

\section{Publisher's Note}

Springer Nature remains neutral with regard to jurisdictional claims in published maps and institutional affiliations.

\section{Received: 25 December 2018 Accepted: 26 February 2019} Published online: 20 March 2019

\section{References}

1. Katayama R, Maezawa T. Total replacement of the knee joint by prosthesis. Nihon Seikeigeka Gakkai Zasshi. 1968;42(3):187-92.

2. Recommendations for the medical management of osteoarthritis of the hip and knee: 2000 update. American College of Rheumatology Subcommittee on Osteoarthritis Guidelines. Arthritis Rheum. 2000;43(9):1905-15.

3. Richmond J, Hunter D, Irrgang J, Jones MH, Snyder-Mackler L, Van Durme D, Rubin C, Matzkin EG, Marx RG, Levy BA, et al. American Academy of Orthopaedic surgeons clinical practice guideline on the treatment of osteoarthritis (OA) of the knee. J Bone Joint Surg Am. 2010;92(4):990-3.

4. Zhang W, Nuki G, Moskowitz RW, Abramson S, Altman RD, Arden NK, Bierma-Zeinstra S, Brandt KD, Croft P, Doherty M, et al. OARSI recommendations for the management of hip and knee osteoarthritis: part III: changes in evidence following systematic cumulative update of research published through January 2009. Osteoarthr Cartil. 2010;18(4):476-99.

5. Anakwe RE, Jenkins PJ, Moran M. Predicting dissatisfaction after total hip arthroplasty: a study of 850 patients. J Arthroplast. 2011;26(2):209-13.

6. Bourne RB, Chesworth BM, Davis AM, Mahomed NN, Charron KD. Patien satisfaction after total knee arthroplasty: who is satisfied and who is not? Clin Orthop Relat Res. 2010;468(1):57-63.

7. Font-Rodriguez DE, Scuderi GR, Insall JN. Survivorship of cemented total knee arthroplasty. Clin Orthop Relat R. 1997;345:79-86.

8. Pavone V, Boettner F, Fickert S, Sculco TP. Total condylar knee arthroplasty: a long-term followup. Clin Orthop Relat R. 2001;388:18-25.

9. Kelly MA, Clarke HD. Long-term results of posterior cruciate-substituting total knee arthroplasty. Clin Orthop Relat R. 2002:404:51-7.

10. Ranawat CS, Flynn WFJ, Saddler S, Hansraj KK, Maynard MJ. Long-term results of the Total condylar knee arthroplasty: a 15-year survivorship study. Clin Orthop Relat R. 1993:(286):94-102.

11. Anderson JG, Wixson RL, Tsai D, Stulberg SD, Chang RW. Functional outcome and patient satisfaction in total knee patients over the age of 75. J Arthroplast. 1996;11(7):831-40.

12. Tria AJ, Coon TM. Minimal incision total knee arthroplasty: early experience. Clin Orthop Relat Res. 2003;416:185-90.

13. Niki Y, Mochizuki T, Momohara S, Saito S, Toyama Y, Matsumoto H. Is minimally invasive surgery in total knee arthroplasty really minimally invasive surgery? J Arthroplast. 2009;24(4):499-504.

14. Huang $A B$, Wang $H J$, Yu JK, Yang B, Ma D, Zhang JY. Optimal patellar alignment with minimally invasive approaches in total knee arthroplasty after a minimum five year follow-up. Int Orthop. 2016:40(3):487-92.

15. Yang JH, Yoon JR, Pandher DS, Oh KJ. Clinical and radiologic outcomes of contemporary 3 techniques of TKA. Orthopedics. 2010;33(10 Suppl):76-81.

16. Karpman RR, Smith HL. Comparison of the early results of minimally invasive vs standard approaches to total knee arthroplasty a prospective randomized study. J Arthroplasty. 2009;24(5):681-8.

17. Chotanaphuti T, Ongnamthip P, Karnchanalerk K, Udombuathong P. Comparative study between $2 \mathrm{~cm}$ limited quadriceps exposure minimal invasive surgery and conventional total knee arthroplasty in quadriceps function: prospective randomized controlled trial. J Med Assoc Thail. 2008; 91(2):203-7.

18. Shen $\mathrm{H}$, Zhang $X \mathrm{~L}$, Wang $\mathrm{Q}$, Shao JJ, Jiang Y. Minimally invasive total knee arthroplasty through a quadriceps sparing approach: a comparative study. Zhonghua Wai Ke Za Zhi. 2007:45(16):1083-6.

19. Huang HT, Su JY, Chang JK, Chen CH, Wang GJ. The early clinical outcome of minimally invasive quadriceps-sparing total knee arthroplasty: report of a 2-year follow-up. J Arthroplast. 2007;22(7):1007-12.
20. King J, Stamper DL, Schaad DC, Leopold SS. Minimally invasive total knee arthroplasty compared with traditional total knee arthroplasty assessment of the learning curve and the postoperative recuperative period. J Bone Joint Surg Am. 2007:89(7):1497-503.

21. Chin PL, Foo LS, Yang KY, Yeo SJ, Lo NN. Randomized controlled trial comparing the radiologic outcomes of conventional and minimally invasive techniques for total knee arthroplasty. J Arthroplast. 2007;22(6):800-6.

22. Tashiro Y, Miura H, Matsuda S, Okazaki K, Iwamoto Y. Minimally invasive versus standard approach in total knee arthroplasty. Clin Orthop Relat Res. 2007:463:144-50.

23. Chen AF, Alan RK, Redziniak DE, Tria AJ. Quadriceps sparing total knee replacement the initial experience with results at two to four years. J Bone Joint Surg Br. 2006;88(11):1448-53.

24. Chiang H, Lee CC, Lin WP, Jiang CC. Comparison of quadriceps-sparing minimally invasive and medial parapatellar total knee arthroplasty: a 2-year follow-up study. J Formos Med Assoc. 2012;111(12):698-704.

25. Kim YH, Kim JS, Kim DY. Clinical outcome and rate of complications after primary total knee replacement performed with quadriceps-sparing or standard arthrotomy. J Bone Joint Surg Br. 2007:89(4):467-70.

26. Kim YH, Sohn KS, Kim JS. Short-term results of primary total knee arthroplasties performed with a mini-incision or a standard incision. J Arthroplast. 2006;21(5):712-8.

27. Moher D, Liberati A, Tetzlaff J, Altman DG. Preferred reporting items for systematic reviews and meta-analyses: the PRISMA statement. BMJ. 2009; 339:b2535.

28. Shamseer L, Moher D, Clarke M, Ghersi D, Liberati A, Petticrew M, Shekelle P, Stewart LA. Preferred reporting items for systematic review and metaanalysis protocols (PRISMA-P) 2015: elaboration and explanation. BMJ. 2015; 349:g7647.

29. Higgins JPT GS. Cochrane handbook for systematic reviews of interventions, vol. 2017. The Cochrane Collaboration; 2011. Available: http://handbook. cochrane.org/front_page.htm.

30. Wells GA SB. The Newcastle-Ottawa scale (NOS) for assessing the quality of nonrandomised studies in meta-analyses, vol. 2017. Available: http://www. ohri.ca/programs/dinical_epidemiology/oxford.asp.

31. Hozo SP, Djulbegovic B, Hozo I. Estimating the mean and variance from the median, range, and the size of a sample. BMC Med Res Methodol. 2005;5:13.

32. Lin SY, Chen CH, Fu YC, Huang PJ, Lu CC, Su JY, Chang JK, Huang HT. Comparison of the clinical and radiological outcomes of three minimally invasive techniques for total knee replacement at two years. Bone Joint J. 2013;95B(7):906-10.

33. J X C-HL, S-G Z YL. Total knee arthroplasty: comparison between quadriceps sparing approach and medial parapatellar approach. Chinese J Tissue Eng Res. 2013;35.

34. Matsumoto T, Muratsu H, Kubo S, Mizuno K, Kinoshita K, Ishida K, Matsushita T, Sasaki K, Tei K, Takayama K, et al. Soft tissue balance measurement in minimal incision surgery compared to conventional total knee arthroplasty. Knee Surg Sports Traumatol Arthrosc. 2011;19(6):880-6

35. Qi YS, Yang B, Yu JK, Zhang JY, Huang AB, Wang HJ. Does quadricepssparing Total knee arthroplasty increase the risk of lower limb and component malalignment? A minimum 5-year follow-up study. Chin Med J. 2016;129(1):92-4.

36. Lee WC, Kwan YH, Chong HC, Yeo SJ. The minimal clinically important difference for knee society clinical rating system after total knee arthroplasty for primary osteoarthritis. Knee Surg Sports Traumatol Arthrosc. 2017;25(11): $3354-9$

37. Danoff JR, Goel R, Sutton R, Maltenfort MG, Austin MS. How much pain is significant? Defining the minimal clinically important difference for the visual analog scale for pain after Total joint arthroplasty. J Arthroplast. 2018; 33(7S):S71-5.

38. Fang DM, Ritter MA, Davis KE. Coronal alignment in total knee arthroplasty: just how important is it? J Arthroplast. 2009;24(6 Suppl):39-43.

39. Ritter MA, Davis KE, Meding JB, Pierson JL, Berend ME, Malinzak RA. The effect of alignment and BMI on failure of total knee replacement. J Bone Joint Surg Am. 2011;93(17):1588-96.

40. Peng $X$, Zhang $X$, Cheng T, Cheng M, Wang J. Comparison of the quadriceps-sparing and subvastus approaches versus the standard parapatellar approach in total knee arthroplasty: a meta-analysis of randomized controlled trials. BMC Musculoskelet Disord. 2015;16:327.

41. Kazarian GS, Siow MY, Chen AF, Deirmengian CA. Comparison of quadriceps-sparing and medial Parapatellar approaches in Total knee 
arthroplasty: a meta-analysis of randomized controlled trials. J Arthroplast. 2018:33(1):277-83.

42. Tasker A, Hassaballa M, Murray J, Lancaster S, Artz N, Harries W, Porteous A. Minimally invasive total knee arthroplasty; a pragmatic randomised

controlled trial reporting outcomes up to 2 year follow up. Knee. 2014;21(1): 189-93.

43. Lin WP, Lin J, Horng LC, Chang SM, Jiang CC. Quadriceps-sparing, minimalincision total knee arthroplasty a comparative study. J Arthroplasty. 2009; 24(7):1024-32.

Ready to submit your research? Choose BMC and benefit from:

- fast, convenient online submission

- thorough peer review by experienced researchers in your field

- rapid publication on acceptance

- support for research data, including large and complex data types

- gold Open Access which fosters wider collaboration and increased citations

- maximum visibility for your research: over $100 \mathrm{M}$ website views per year

At BMC, research is always in progress.

Learn more biomedcentral.com/submissions 